\title{
有机膦酸构筑的多铇氧簇稀土化合物的设计合成、结构 及性质
}

杨宗斐，马鹏涛，王敬平*，牛景杨 ${ }^{*}$

河南大学化学化工学院, 河南省多酸化学重点实验室, 分子与晶体研究所, 开封 475004

*联系人, E-mail: jpwang@ @enu.edu.cn; jyniu@ @enu.edu.cn

2018-06-19 收稿, 2018-07-27 修回, 2018-08-01 接受, 2018-08-17 网络版发表

国家自然科学基金(21571050, 21573056, 21771053, 21771054)资助

摘要在常规水溶液条件下, 选择介稳态的前驱体 $\mathrm{K}_{14}\left[\mathrm{As}_{2} \mathrm{~W}_{19} \mathrm{O}_{67}\left(\mathrm{H}_{2} \mathrm{O}\right)\right]$ 与配体羟基乙叉二膦酸(HEDP) 及稀土 化合物反应, 合成了一例有机膦酸构筑的多铇氧簇稀土化合物 $\mathrm{CsH}\left[\mathrm{Pr}\left(\mathrm{H}_{2} \mathrm{O}\right)_{4}\left(\mathrm{AsW}_{9} \mathrm{O}_{33}\right)\left(\mathrm{W}_{2} \mathrm{O}_{3}\right)\left(\mathrm{O}_{3} \mathrm{PCOHCH}_{3} \mathrm{PO}_{3}\right)\right.$ $\left.\operatorname{Pr}\left(\mathrm{H}_{2} \mathrm{O}\right)_{7}\right]_{2} \cdot 19 \mathrm{H}_{2} \mathrm{O}(\mathbf{1})$, 并通过单晶X射线衍射分析、元素分析、傅里叶红外光谱分析等对其结构进行了表征. 晶体 解析表明, 化合物 $\mathbf{1}$ 由 1 个 $\mathrm{S}$ 形结构框架 $\left\{\left[\left(\mathrm{AsW}_{9} \mathrm{O}_{33}\right)\left(\mathrm{W}_{2} \mathrm{O}_{3}\right)\right]_{2}\right\}, 2$ 个有机磷酸基团 $\left\{\mathrm{O}_{3} \mathrm{PCOHCH}_{3} \mathrm{PO}_{3}\right\}$ 和 4 个 $\operatorname{Pr}^{3+}$ 离子通 过一定的方式键连形成三维框架结构。磁学性质研究表明, 化合物 $\mathbf{1}$ 呈现出反铁磁性耦合作用。荧光性质研究表 明，化合物1在近红外区呈现出双寿命发光，其中 $\tau_{1}=4.76 \mu \mathrm{s}(35.53 \%), \tau_{2}=9.71 \mu \mathrm{s}(64.4 \%)$.

关键词多金属氧簇, 有机膦酸, 晶体结构, 稀土

多金属氧酸盐(POMs)由于其阴离子结构的多样 性及良好的理化性质, 在光、电、磁材料及催化方面 具有广阔的应用前景, 受到人们的广泛关注 ${ }^{[1-5]}$. 有 机膦酸结构多样、种类繁多, 并且具有高熔点、可修 饰、丰富的键合方式等特性, 在材料、医药和催化等 领域具有潜在应用价值. 因此, 将多金属氧酸盐和有 机膦酸结合起来, 有望构筑结构新颖、性能优良的有 机-无机杂化化合物, 逐渐成为多酸化学的研究热点 之一. 1994年, Pope课题组 ${ }^{[6]}$ 合成了首例有机膦酸构 筑的多铇氧簇, 其阴离子结构为 $\left[\left(\mathrm{O}_{3} \mathrm{PCH}_{2} \mathrm{PO}_{3}\right)_{4}\right.$ $\left.\mathrm{W}_{12} \mathrm{O}_{36}\right]^{16-}$. 在1998和1999年, Thouvenot课题组报道 了几例有机磷酸构筑的多铇氧簇 $\left\{\mathrm{PW}_{9} \mathrm{O}_{34}(\mathrm{RPO})_{2}\right\}$ (R=Et, $n$-Bu, $t$-Bu烯丙基, $\mathrm{Ph})^{[7]}$ 和 $\left\{\mathrm{SiW}_{10} \mathrm{O}_{36}(\mathrm{RPO})_{2}\right\}$ $\left(\mathrm{R}=\mathrm{Et}, n-\mathrm{Bu}, t-\mathrm{Bu}, \mathrm{C}_{2} \mathrm{H}_{4} \mathrm{COOH}, \mathrm{Ph}\right)^{[8]}$. 其后许多基于 缺位Keggin和Lindqvist结构的有机磷酸修饰的多铇氧 簇被报道, 比如: $\left\{\left(\mathrm{SiW}_{10} \mathrm{O}_{36}\right)\left[\mathrm{O}=\mathrm{PCH}_{2}\left(\mathrm{C}_{6} \mathrm{H}_{4}\right)_{\mathrm{n}} \mathrm{CH}_{2} \mathrm{P}=\right.\right.$
$\mathrm{O}]\}_{2}(n=1,2)^{[9]},\left\{\mathrm{NbW}_{10} \mathrm{O}_{38}(\mathrm{RP})_{2}\right\}(\mathrm{R}=\mathrm{Me}, \mathrm{Et}, \mathrm{Pr}, n \mathrm{Bu}$, 己基，庚基，环已基， $\mathrm{Ph}$, 烯丙基 ${ }^{[10]},\left\{(\mathrm{R}-\mathrm{PO})_{2}\right.$ $\left.\left(\gamma-\mathrm{XW}_{10} \mathrm{O}_{36}\right)\right\}(\mathrm{X}=\mathrm{Si}, \mathrm{Ge} ; \mathrm{R}=$ 氮被保护的氨基烷基或氧 被保护的氨基酸衍生物 $)^{[11]},\left\{\left[\left(\mathrm{B}-\alpha-\mathrm{PW}_{9} \mathrm{O}_{34}\right) \mathrm{Co}_{3}(\mathrm{OH})\right.\right.$ $\left.\left.\left(\mathrm{H}_{2} \mathrm{O}\right)_{2}\left(\mathrm{O}_{3} \mathrm{PC}(\mathrm{O})\left(\mathrm{C}_{3} \mathrm{H}_{6} \mathrm{NH}_{3}\right) \mathrm{PO}_{3}\right)\right]_{2} \mathrm{Co}\right\}^{[12]},\left\{\left[\left(\mathrm{BPW}_{9} \mathrm{O}_{34}\right)\right.\right.$ $\left.\left.\mathrm{Ni}_{3}(\mathrm{OH})\left(\mathrm{H}_{2} \mathrm{O}\right)_{2}(\mathrm{Ale})\right]_{2} \mathrm{Ni}\right\}\left(\mathrm{Ale}=\mathrm{H}_{2} \mathrm{O}_{3} \mathrm{PC}\left(\mathrm{C}_{3} \mathrm{H}_{6} \mathrm{~N}-\mathrm{H}_{2}\right)(\mathrm{OH})\right.$ $\left.\mathrm{PO}_{3} \mathrm{H}_{2}\right)^{[13]},\left\{\mathrm{AsW}_{9} \mathrm{O}_{33}\{\mathrm{P}(\mathrm{O}) \mathrm{R}\}_{2}\right\}\left(\mathrm{R}=\mathrm{CH}_{2} \mathrm{CH}_{2} \mathrm{COOH}, \mathrm{CH}_{2}-\right.$ $\left.\mathrm{CH}_{2} \mathrm{CONHCH}_{2} \mathrm{C}_{6} \mathrm{H}_{5}\right)^{[14]}$ 和 $\left\{\mathrm{H}_{4}\left(\mathrm{AsW}_{9} \mathrm{O}_{33}\right)_{2}\left[\mathrm{TM}\left(\mathrm{H}_{2} \mathrm{O}\right) \mathrm{W}_{5^{-}}\right.\right.$ $\left.\left.\mathrm{O}_{11}\left(\mathrm{~N}\left(\mathrm{CH}_{2} \mathrm{PO}_{3}\right)_{3}\right)\right]_{2}\left(\mu_{2}-\mathrm{O}\right)_{2}\right\}(\mathrm{TM}=\mathrm{Zn}, \mathrm{Ni}, \mathrm{Co}, \mathrm{Mn})^{[15,16]}$, 已报道的该类化合物大多呈现孤立结构, 并且大多 数的化合物只含有过渡金属离子或者没有含额外的 金属离子. 相比较而言, 有机膦酸构筑的多铇氧簇稀 土化合物则非常少见, 目前只有本课题组 ${ }^{[17]}$ 于 2017 年报道的 4 例同构化合物, 它们的分子式为 $\mathrm{K}_{2}[\mathrm{Ln}-$ $\left.\left(\mathrm{H}_{2} \mathrm{O}\right)_{4}\left(\mathrm{AsW}_{9} \mathrm{O}_{33}\right)\left(\mathrm{W}_{2} \mathrm{O}_{3}\right)\left(\mathrm{O}_{3} \mathrm{PCOHCH}_{3}-\mathrm{PO}_{3}\right) \mathrm{Ln}\left(\mathrm{H}_{2} \mathrm{O}\right)_{7}\right]_{2}$. $15 \mathrm{H}_{2} \mathrm{O}(\mathrm{Ln}=\mathrm{Ce}, \mathrm{Nd}, \mathrm{Sm}, \mathrm{Eu})$. 稀土元素因其特有的 $4 \mathrm{f}$ 
电子, 使其具有良好的光、电、磁性质, 所以将稀土 元素引人到有机膦酸构筑的多铇氧簇阴离子的骨架 中, 不仅可以构筑结构新颖的化合物, 而且可使化合 物具有优良的苂光和磁学等性质. 这使得我们对探 索稀土离子参与构筑有机膦酸功能化的多铇氧簇产生 了极大的兴趣. 本文通过引人抗衡阳离子 $\mathrm{Cs}^{+}$, 运用常 规水溶液法得到了 1 例有机膦酸构筑的多铇氧簇稀土 化合物 $\mathrm{CsH}\left[\mathrm{Pr}\left(\mathrm{H}_{2} \mathrm{O}\right)_{4}\left(\mathrm{AsW}_{9} \mathrm{O}_{33}\right)\left(\mathrm{W}_{2} \mathrm{O}_{3}\right)\left(\mathrm{O}_{3} \mathrm{PCOHCH}_{3}-\right.\right.$ $\left.\left.\mathrm{PO}_{3}\right) \operatorname{Pr}\left(\mathrm{H}_{2} \mathrm{O}\right)_{7}\right]_{2} \cdot 19 \mathrm{H}_{2} \mathrm{O}(\mathbf{1})$.

\section{1 实验}

( i ) 试剂和仪器. 前驱体 $\mathrm{K}_{14}\left[\mathrm{As}_{2} \mathrm{~W}_{19} \mathrm{O}_{67}\left(\mathrm{H}_{2} \mathrm{O}\right)\right]$ 根据文献[18]合成并通过红外表征, 其余试剂为商业 购买的分析纯试剂, 使用时未经进一步纯化. 红外光 谱用 Bruker VERTEX 70 IR型傅立叶红外光谱仪测 定, 记录范围为 $4000 \sim 450 \mathrm{~cm}^{-1}, \mathrm{KBr}$ 压片. 热分析数 据用Metter-Toledo TGA/SDTA851 $1^{\mathrm{e}}$ 型热重分析仪在 $\mathrm{N}_{2}$ 氛围中测量. $\mathrm{C}, \mathrm{H}, \mathrm{N}$ 元素分析使用Perkin-Elmer $240 \mathrm{C}$ 型元素分析仪测定. X射线粉末衍射数据使用 Bruker AXS D8 Advance粉末衍射仪测定(射线源为 $\mathrm{Cu}$ K $\alpha$ 射线, $\lambda=1.54056 \AA, 2 \theta=5^{\circ} \sim 40^{\circ}$ ). 磁学性质利 用美国Quantum Design MPMS3 SQUID系统测定 $(H=$ $1000 \mathrm{Oe}, T=1.8 \sim 300 \mathrm{~K})$. 苂光光谱使用英国 EDINBURGH FLS 980型瞬态稳态苂光光谱仪测定.

(ii) 化合物 $\mathbf{1}$ 的合成. 将 $\mathrm{K}_{14}\left[\mathrm{As}_{2} \mathrm{~W}_{19} \mathrm{O}_{67}\left(\mathrm{H}_{2} \mathrm{O}\right)\right]$ $(0.66 \mathrm{~g}, 0.125 \mathrm{mmol})$ 和羟基乙叉二膦酸 (HEDP, $0.11 \mathrm{~g}$, $0.36 \mathrm{mmol}$ )溶解于 $15 \mathrm{~mL}$ 蒸馏水中, 搅拌至澄清后加
人 $\mathrm{PrCl}_{3} \cdot 6 \mathrm{H}_{2} \mathrm{O}(0.107 \mathrm{~g}, 0.30 \mathrm{mmol})$, 之后滴加 $3 \mathrm{~mol} / \mathrm{L}$ $\mathrm{KOH}$ 调节 $\mathrm{pH} 1.30$, 将此溶液置于 $60^{\circ} \mathrm{C}$ 水浴中搅拌下 反应 $1 \mathrm{~h}$, 取出溶液趁热加人 $0.1 \mathrm{~g} \mathrm{CsCl}$. 冷却至室温, 过滤后滤液室温下缓慢挥发, 大约 2 周后得到绿色针 状晶体. 产率: $0.205 \mathrm{~g}\left(37.89 \%\right.$, 以 $\mathrm{PrCl}_{3} \cdot 6 \mathrm{H}_{2} \mathrm{O}$ 计). 元 素分析 (\%): 理论值: C, $0.67 ; \mathrm{H}, 1.28$; 实验值: C, 0.67; H, 1.21. IR $\left(v, \mathrm{~cm}^{-1}\right)$ : 3419(br), 1616(s), 1168(s), 1085(m), 968(s), 868(s), 768(s), 668(s). 在合成中尝试 将 $\mathrm{Cs}^{+}$离子替换为其他种类的阳离子, 如 $\mathrm{K}^{+}, \mathrm{Rb}^{+}$, $\mathrm{TMA}^{+}$, 通过大量对比实验发现都无法获得此化合 物. 由此推测, 抗衡阳离子的尺寸、电荷、对称性及 溶解性等可能对多酸阴离子的结晶、构型、原子配位 有一定影响 ${ }^{[19 \sim 21]}$.

(iii) $\mathrm{X}$ 射线晶体结构测定. 选取大小为 $0.23 \times$ $0.18 \times 0.15$ 的绿色针状晶体, 置于Bruker Apex-II CCD 衍射仪上, 采用石墨单色器的Mo K $\alpha$ 射线 $(\lambda=0.71073$ $\AA$ )为辐射源, 以 $\omega-2 \theta$ 扫描方式收集衍射数据. 在 296(2) K温度下, $3.25^{\circ}<\theta<50.2^{\circ}$ 的范围内共收集 30761 个衍射点. 晶体结构通过直接法解出, 全部数 据经 $L \mathrm{p}$ 因子和经验吸收校正. 全部计算工作均使用 SHELXTL-97程序系统在计算机上完成. 晶体数据的 CCDC号为708379.

\section{2 结果与讨论}

\section{1 晶体结构}

$\mathrm{X}$ 射线单晶结构分析表明，化合物 $\mathbf{1}$ 属于单斜晶

表 1 化合物 1 的主要晶体学数据

Table 1 Crystal data and structure refinements for compound 1

\begin{tabular}{|c|c|c|c|}
\hline \multicolumn{2}{|c|}{ 化合物 1 的主要晶体学数据 } & \multicolumn{2}{|c|}{ 化合物 1 的主要晶体学数据 } \\
\hline 分子式 & $\mathrm{C}_{4} \mathrm{H}_{91} \mathrm{P}_{4} \mathrm{O}_{127} \mathrm{As}_{2} \mathrm{Pr}_{4} \mathrm{~W}_{22} \mathrm{Cs}$ & 单胞中电子的数目 & 6202.0 \\
\hline 分子量 & 7186.44 & 晶体尺寸 $\left(\mathrm{mm}^{3}\right)$ & $0.23 \times 0.18 \times 0.15$ \\
\hline 衍射实验温度(K) & $296(2)$ & 最大与最小衍射指标 & $\begin{array}{lll}-17 & h & 18\end{array}$ \\
\hline $\begin{array}{l}\text { 空间群 } \\
\text { 晶系 }\end{array}$ & $\begin{array}{l}P 2{ }_{1} / c \\
\text { 单斜 }\end{array}$ & & $\begin{array}{lll}-18 & k & 17 \\
-30 & l & 31\end{array}$ \\
\hline$a(\AA)$ & $15.1101(6)$ & 衍射角 $\left(^{\circ}\right)$ & $3.25 \sim 50.2$ \\
\hline$b(\AA)$ & $15.8956(7)$ & 总衍射点数目 & 30761 \\
\hline$c(\AA)$ & $26.1615(11)$ & 等效点平均标准偏差 & 0.0526 \\
\hline$\beta\left(^{\circ}\right)$ & 106.6120(10) & 拟合优度 & 1.024 \\
\hline 晶胞体积 $\left(\AA^{3}\right)$ & $6021.3(4)$ & 对于可观测衍射点的最终残差因子 & $\begin{array}{l}R_{1}=0.0428 \\
w R_{2}=0.0996\end{array}$ \\
\hline 单胞中分子式的数量 & 2 & 对于全部衍射点的最终残差因子 & $\begin{array}{l}R_{1}=0.0626 \\
w R_{2}=0.1093\end{array}$ \\
\hline 衍射实验计算获得的晶胞密度 $\left(\mathrm{g} \mathrm{cm}^{-3}\right)$ & 3.892 & & \\
\hline
\end{tabular}


系, $P 2{ }_{1} / c$ 空间群. 价键计算结果表明 ${ }^{[22]}$, 该化合物中 所有 $\mathrm{W}, \mathrm{As}, \mathrm{P}$ 和 $\mathrm{Pr}$ 原子的化合价依次为 $+6,+3,+3$ 和 +3. 该化合物包含 19 个结晶水分子, 1 个 $\mathrm{Cs}^{+}$离子, 1 个 $\mathrm{H}^{+}$和 1 个聚阴离子 $\left\{\left[\mathrm{Pr}\left(\mathrm{H}_{2} \mathrm{O}\right)_{4}\left(\mathrm{AsW}_{9} \mathrm{O}_{33}\right)\left(\mathrm{W}_{2} \mathrm{O}_{3}\right)\left(\mathrm{O}_{3}-\right.\right.\right.$ $\left.\left.\left.\mathrm{PCOHCH}_{3} \mathrm{PO}_{3}\right) \operatorname{Pr}\left(\mathrm{H}_{2} \mathrm{O}\right)_{7}\right]_{2}\right\}^{2-}$. 化合物 $\mathbf{1}$ 的聚阴离子可 以看作 3 步形成: (1) 2 个 $\left\{\mathrm{AsW}_{9} \mathrm{O}_{33}\right\}$ 亚单元通过 1 对 $\left\{\mathrm{W}_{2} \mathrm{O}_{11}\right\}$ 片段连接形成 $\mathrm{S}$ 形结构框架 $\left\{\left[\left(\mathrm{AsW}_{9} \mathrm{O}_{33}\right)\right.\right.$ $\left.\left.\left(\mathrm{W}_{2} \mathrm{O}_{3}\right)\right]_{2}\right\}$ (图 1(c)); (2) 2 个 Pr1离子分别通过4个Pr1$\mathrm{O}-(\mathrm{W})$ 共价键与 $\mathrm{S}$ 形结构框架连接, 形成 $\left\{\left[\mathrm{Pr}\left(\mathrm{H}_{2} \mathrm{O}\right)_{4}\left(\mathrm{AsW}_{9} \mathrm{O}_{33}\right)\left(\mathrm{W}_{2} \mathrm{O}_{3}\right)\right]_{2}\right\}$ 单元(图 1(d)); (3) 2 个 $\left\{\left(\mathrm{O}_{3} \mathrm{PCOHCH}_{3} \mathrm{PO}_{3}\right) \operatorname{Pr}\left(\mathrm{H}_{2} \mathrm{O}\right)_{7}\right\}$ 单元分别通过 4 个 $\mathrm{P}-\mathrm{O}-(\mathrm{W})$ 共价键与 $\left\{\left[\mathrm{Pr}\left(\mathrm{H}_{2} \mathrm{O}\right)_{4}\left(\mathrm{AsW}_{9} \mathrm{O}_{33}\right)\left(\mathrm{W}_{2} \mathrm{O}_{3}\right)\right]_{2}\right\}$ 单 元连接, 形成聚阴离子, 如图 1(e)所示. 该聚阴离子 通过 $(\mathrm{W})-\mathrm{O}-\mathrm{Pr} 1-\mathrm{O}-(\mathrm{W})$ 连接形成一维链状结构, 同 时通过 $(\mathrm{P})-\mathrm{O}-\mathrm{Pr} 2-\mathrm{O}-(\mathrm{P})$ 与 4 个相同的阴离子相连无 限延伸形成二维平面结构. 经过 $\operatorname{Pr} 1, \operatorname{Pr} 2$ 离子和有机 膦酸片段的协同延伸, 化合物 $\mathbf{1}$ 的三维堆积图 (图1(f)).

在化合物 1 中, 2 个晶体学独立的 $\operatorname{Pr}$ 离子都是九配 位的单加冠四方反棱柱构型. 对于 $\operatorname{Pr} 1$ 来说(图 1(g)), 单加冠的位置被 $\mathrm{O} 2 \mathrm{~W}$ 占据, 四方反棱柱的 2 个底面分 别由 O5, O1W, O3W, O4W和O1, O2, O29, O40构成. $\operatorname{Pr} 1$ 离子与 4 个水分子和 5 个二重桥氧配位, 化学键键
长范围是 $\operatorname{Pr} 1-\mathrm{O}\left(\mathrm{H}_{2} \mathrm{O}\right)$ : 2.514(16) 2.550(16) $\AA$ 和 Pr1- $\mu_{2}-\mathrm{O}: 2.495(10) 2.517(10) \AA$. 对于 $\operatorname{Pr} 2$ 来说 (图 $1(\mathrm{f})$ ), 单加冠的位置被 $\mathrm{O} 26$ 占据, 2 个底面分别由 O5W, O6W, O8W, O11W和O28, O7W, O9W, O10W 构成. $\operatorname{Pr} 2$ 离子与 7 个水分子配位, 化学键键长范围是 $\operatorname{Pr} 2-\mathrm{O}\left(\mathrm{H}_{2} \mathrm{O}\right)$ : 2.491(16) 2.641(19) $\AA$, 另外2个配位点 为二重桥氧原子, 对应的键长分别为 Pr2-O26:

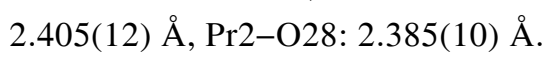

\section{2 红外光谱, 热重分析及 $X$ 射线粉未衍射}

化合物 1 的红外光谱如图 2(a)所示, 出现在 768, 868 和 $968 \mathrm{~cm}^{-1}$ 位置的特征振动峰分别归属于 $v\left(\mathrm{~W}-\mathrm{O}_{\mathrm{b}}\right), v\left(\mathrm{As}-\mathrm{O}_{\mathrm{a}}\right)$ 和 $v\left(\mathrm{~W}-\mathrm{O}_{\mathrm{t}}\right)$ 的伸缩振动 ${ }^{[23]} .1168$ 和 $1085 \mathrm{~cm}^{-1}$ 位置的特征振动峰可归属为 HEDP中 P-O键 的伸缩振动, 而 HEDP中P-C的伸缩振动峰出现在的 $668 \mathrm{~cm}^{-1}$ 附近 ${ }^{[24]}$. 此外, 化合物 $\mathbf{1}$ 在 $3419 \mathrm{~cm}^{-1}$ 附近的 宽强信号峰和 $1616 \mathrm{~cm}^{-1}$ 处的信号峰可分别归属于化 合物中结晶水以及配位水的 $v(\mathrm{O}-\mathrm{H})$ 的伸缩振动和 $\delta(\mathrm{O}-\mathrm{H})$ 的弯曲振动 ${ }^{[23]}$.

如图 2(b)所示, 化合物 $\mathbf{1}$ 的热重分析在 $\mathrm{N}_{2}$ 气氛保 护下, 以 $10^{\circ} \mathrm{C} \mathrm{min}^{-1}$ 的升温速率在 $25 \sim 800^{\circ} \mathrm{C}$ 范围内测 定, 总失重量为 $14.77 \%$ (理论值: $14.51 \%$ ). 在 $25 \sim 80^{\circ} \mathrm{C}$ 之间第1步失重约 $10.53 \%$ (理论值: $10.28 \%$ ), 对应于
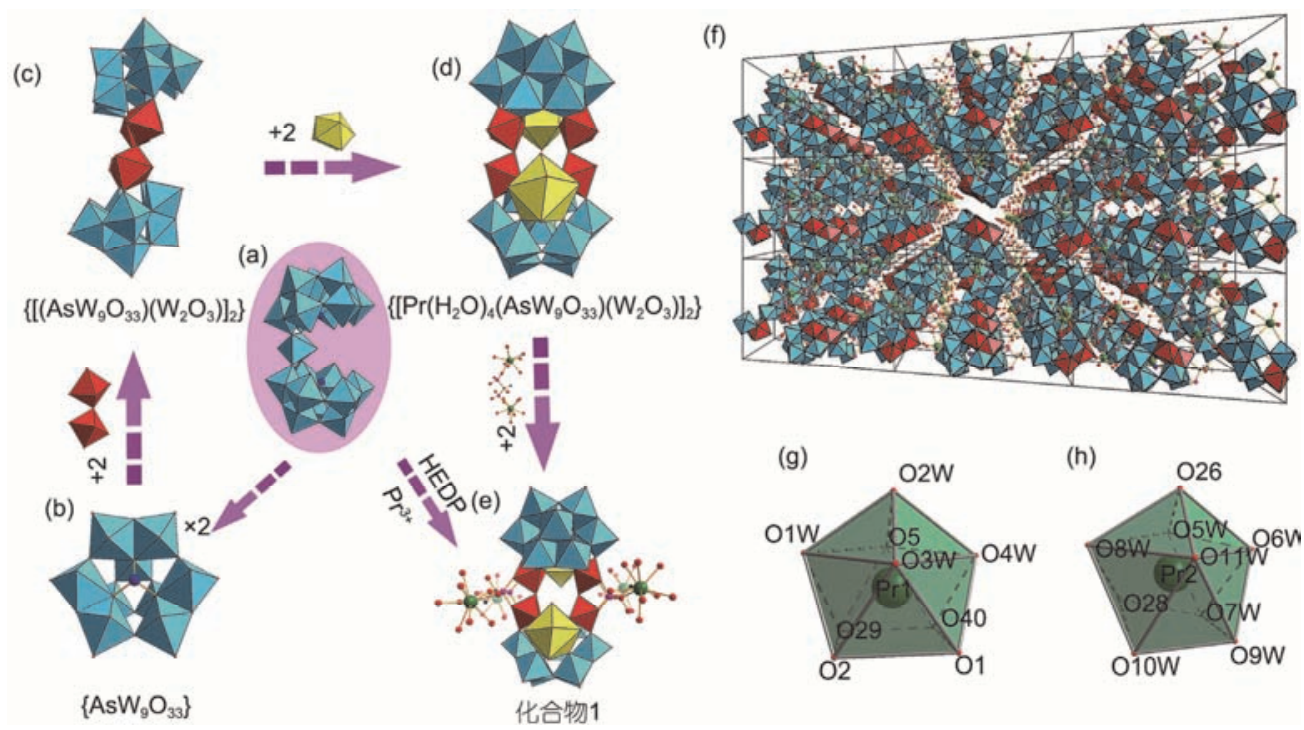

图 1 化合物 1 的结构. (a) (e) 化合物 1 的组装示意图 $\left\{\mathrm{WO}_{6}\right\}$ : 天蓝色和红色的八面体; $\left\{\mathrm{PrO}_{9}\right\}$ : 金色的单加冠四方反棱柱体; Pr: 绿色; P: 紫 红色; As: 深蓝色; C: 灰色; O: 红色; 为清晰可见, 略去氢原子. (f) 化合物 1 的三维堆积图. (g) (h) Pr1 和Pr2 离子的配位环境

Figure 1 The structure of compound 1. (a)-(e) The assembly diagram of compound 1. Color code, $\{$ WO6 $\}$, sky-blue and red octahedral; $\{$ PrO 9$\}$, gold monocapped squareantiprism; Pr, green ball; P, fuchsia ball; As, dark-blue ball; C, gray ball; O, red ball; H atoms are omitted for clarity. (f) The 3D framework of compound 1. (g)-(h) The coordination environment of Pr1 and Pr2 ions 

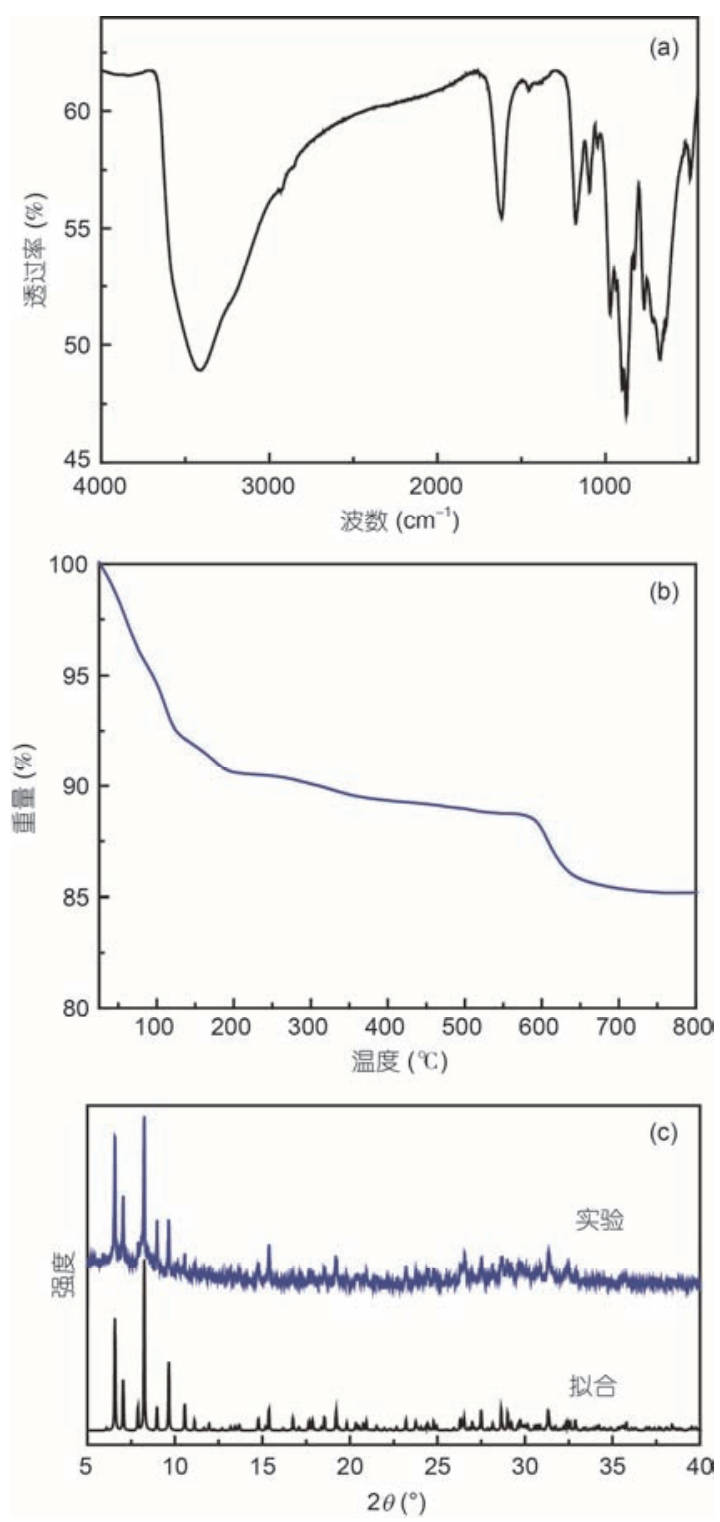

图 2 化合物 1 的红外曲线(a)、热重曲线(b)和粉末衍射图(c)

Figure 2 The IR spectra (a), thermogravimetric curve (b), and XRPD patterns for experiment (top) and simulation (bottom) (c) of compound 1

19 个结晶水分子和 22 个配位水分子的失去. 在 380 $800{ }^{\circ} \mathrm{C}$ ，第2步失重约 $4.24 \%$ (理论值 $4.23 \%$ ), 对应于 2 个有机片段 $\left\{-\mathrm{COHCH}_{3}\right\}$ 的失去, $\mathrm{As}_{2} \mathrm{O}_{3}$ 分子的升华及 1 个结构水的失去 ${ }^{[16]}$.

如图 2(c)所示，化合物 $\mathbf{1}$ 的实测与理论 $X$ 射线粉 末衍射图谱吻合较好, 说明化合物1样品是纯净的.

\section{3 磁学性质}

在1.8 300 K温度范围内, 对化合物1进行了磁学 性质的测试. 如图3(a)所示, 室温时, 化合物 $\mathbf{1}$ 的摩尔磁
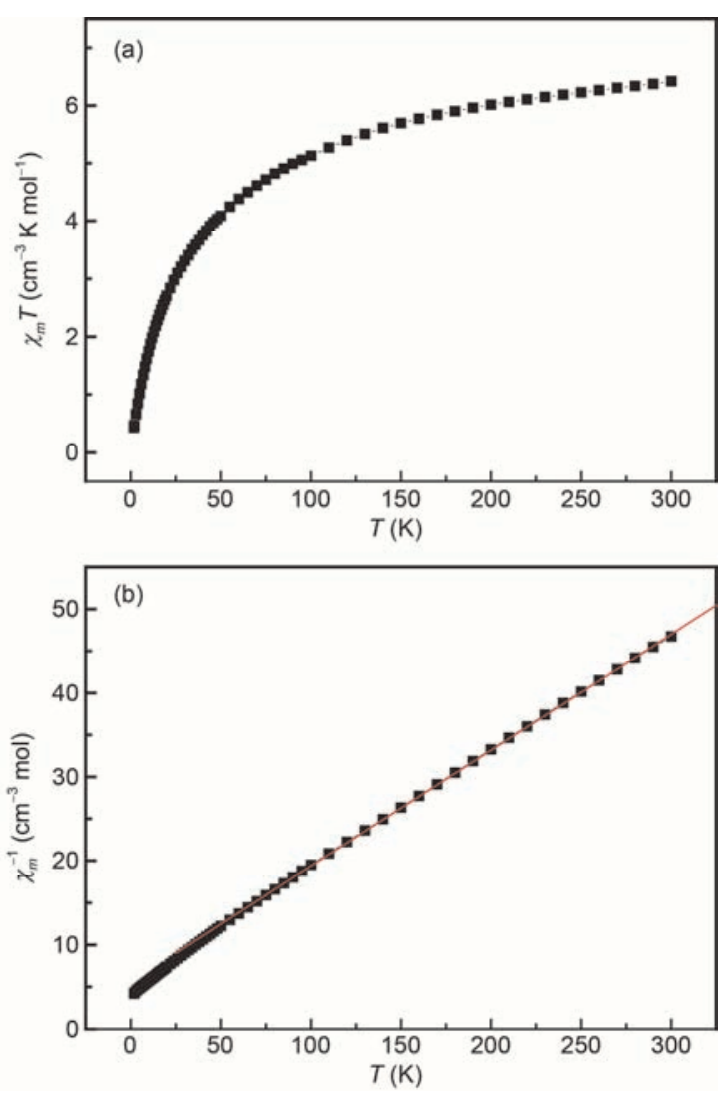

图 3 化合物 1 的 $\chi_{\mathrm{m}} T$ 随 $T$ 变化的曲线(a)和 $1 / \chi_{\mathrm{m}}$ 随 $T$ 变化的曲线(b) Figure $3 \chi_{\mathrm{m}} T$ versus $T$ curves (a) and $1 / \chi_{\mathrm{m}}$ versus $T$ curves for compound 1

化率与绝对温度的乘积 $\chi_{\mathrm{m}} T$ 值为 $6.42 \mathrm{~cm}^{3} \mathrm{~K} \mathrm{~mol}^{-1}$, 与 4 个无相互耦合作用的 $\mathrm{Pr}^{\mathrm{III}}$ 离子理论值 $6.40 \mathrm{~cm}^{3} \mathrm{~K} \mathrm{~mol}^{-1}$ 非常接近 (角量子数 $L=5$, 光谱支项为 ${ }^{3} \mathrm{H}_{4}$, 朗德因子 $g=4 / 5$, 自旋量子数 $S=1$ ). 随测试温度的下降, $\chi_{m} T$ 缓慢 减小, 在温度低于 $101 \mathrm{~K}$ 时, $\chi_{\mathrm{m}} T$ 值随温度的降低而迅 速减小, 在 $1.8 \mathrm{~K}$ 时, 达到最小值 $0.43 \mathrm{~cm}^{3} \mathrm{~K} \mathrm{~mol}^{-1}$. 这 可能是因 $P r$ 离子激发态电子的去布居作用引起的. 此外，如图3(b)所示，在68 300 K温度范围内，通过 居里-外斯定律拟合化合物 $\mathbf{1}$ 的 $1 / \chi_{m}$ 随温度的变化曲 线, 结果表明居里常数 $C=7.20 \mathrm{~cm}^{3} \mathrm{~K} \mathrm{~mol}^{-1}$, 外斯常 数 $\theta=-39.02 \mathrm{~K}$. 结果表明, 化合物 $\mathbf{1}$ 呈现出反铁磁性 耦合作用.

\section{4 苂光性质}

对化合物 $\mathbf{1}$ 的固态粉末室温下的近红外区苂光性 质进行了研究, 结果如图4所示. 化合物 $\mathbf{1}$ 在 $448 \mathrm{~nm}$ 的 激发波长下, 于 $871,1017,1515 \mathrm{~nm}$ 处出现 3 个特征发 射峰, 分别归属为 ${ }^{1} \mathrm{D}_{2} \rightarrow{ }^{3} \mathrm{~F}_{2},{ }^{1} \mathrm{D}_{2} \rightarrow{ }^{3} \mathrm{~F}_{4}$ 和 ${ }^{1} \mathrm{D}_{2} \rightarrow{ }^{1} \mathrm{G}_{4}$ 的光 

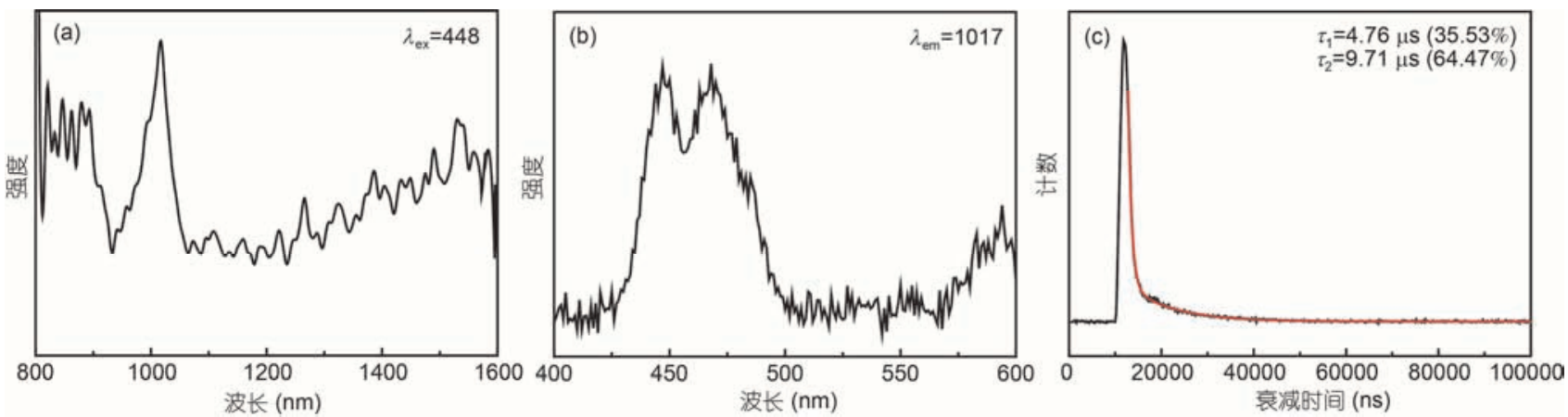

图 4 化合物 $\mathbf{1}$ 的近红外发射光谱(激发波长为 $448 \mathrm{~nm}$ )(a)、近红外激发光谱(b)和近红外区苂光寿命(c)

Figure 4 The NIR emission spectrum (upon $448 \mathrm{~nm}$ excitation) (a), NIR excitation spectra (b), and NIR lifetime decay curve (c) of compound 1

谱跃迁 ${ }^{[25]}$. 此外, 通过监测 $1017 \mathrm{~nm}$ 发射峰和448 nm 激发峰, 测得化合物 $\mathbf{1}$ 的寿命曲线, 该寿命曲线遵循 双指数函数 $I=\mathrm{A}_{1} \exp \left(t / \tau_{1}\right)+\mathrm{A}_{2} \exp \left(t / \tau_{2}\right)$, 其中 $t$ 为某一时 刻, $I$ 为某时刻 $t$ 时的苂光强度, $\mathrm{A}_{1}$ 和 $\mathrm{A}_{2}$ 为加权参数, $\tau_{1}$ 为短寿命, $\tau_{2}$ 为长寿命, 拟合得到苂光寿命 $\tau_{1}=4.76$ $\mu \mathrm{s}(35.53 \%), \tau_{2}=9.71 \mu \mathrm{s}(64.47 \%)$.

\section{3 结论}

本文采用常规水溶液法, 选择HEDP作为配体, 与介稳态的前驱体 $\mathrm{K}_{14}\left[\mathrm{As}_{2} \mathrm{~W}_{19} \mathrm{O}_{67}\left(\mathrm{H}_{2} \mathrm{O}\right)\right]$ 反应, 同时
引人稀土离子, $\mathrm{pH} 1.30$ 的条件下制备了一例有机膦 酸构筑的多铇氧簇稀土化合物, 并对该化合物进行 了磁学性质和苂光性质的研究. 磁学性质研究表明, 化合物 1 呈现出反铁磁性湡合作用. 菼光性质研究表 明，化合物 $\mathbf{1}$ 呈现出双寿命发光. 后续工作将通过选 择不同的前驱体, 其他种类的有机膦酸作配体, 引入 稀土或过渡金属离子，调控抗衡阳离子，以期构筑出 其他结构新预且功能优良的化合物, 系统研究合成 化合物的磁学性质和苂光性质, 并探索化合物的催 化性能.

\section{参考文献}

1 Katsoulis D E. A survey of applications of polyoxometalates. Chem Rev, 1998, 98: 359-388

2 Marrot J, Pilette M A, Haouas M, et al. Polyoxometalates paneling through $\left\{\mathrm{Mo}_{2} \mathrm{O}_{2} \mathrm{~S}_{2}\right\}$ coordination: Cation-directed conformations and chemistry of a supramolecular hexameric scaffold. J Am Chem Soc, 2012, 134: 1724-1737

3 Hill C L. Progress and challenges in polyoxometalate-based catalysis and catalytic materials chemistry. J Mol Catal A Chem, 2007, 262: 2-6

4 Li Z, Li X X, Yang T, et al. Four-shell polyoxometalates featuring high-nuclearity $\ln _{26}$ clusters: Structural transformations of nanoclusters into frameworks triggered by transition-metal ions. Angew Chem Int Ed, 2017, 56: 2664-2669

5 Jin L, Zhu Z K, Wu Y L, et al. Record high-nuclearity polyoxoniobates: Discrete nanoclusters $\left\{\mathrm{Nb}_{114}\right\},\left\{\mathrm{Nb}_{81}\right\}$, and $\left\{\mathrm{Nb}_{52}\right\}$, and extended frameworks based on $\left\{\mathrm{Cu}_{3} \mathrm{Nb}_{78}\right\}$ and $\left\{\mathrm{Cu}_{4} \mathrm{Nb}_{78}\right\}$. Angew Chem Int Ed, 2017, 56: 16288-16292

6 Kortz U, Jameson G B, Pope M T. Polyoxometalate diphosphate complexes. Folded macrocyclic dodecatungstates, $\left[\left(\mathrm{O}_{3} \mathrm{PXPO}_{3}\right)_{4} \mathrm{~W}_{12} \mathrm{O}_{36}\right]$ ${ }^{16-}\left(\mathrm{X}=\mathrm{O}, \mathrm{CH}_{2}\right)$. J Am Chem Soc, 1994, 116: 2659-2660

7 Mayer C R, Thouvenot R. Organophosphoryl derivatives of trivacant tungstophosphates of general formula $\gamma-\mathrm{A}-\left[\mathrm{PW} \mathrm{O}_{34}\left(\mathrm{RPO}_{2}\right]^{5-}: \mathrm{Synthe-}\right.$ sis and structure determination by multinuclear magnetic resonance spectroscopy $\left({ }^{31} \mathrm{P},{ }^{183} \mathrm{~W}\right)$. J Chem Soc Dalton Trans, 1998, (1): 7-14

8 Mayer C R, Herson P, Thouvenot R. Organic-inorganic hybrids based on polyoxometalates. 5. ${ }^{1}$ Synthesis and structural characterization of bis(organophosphoryl) decatungstosilicates $\left[\gamma-\mathrm{SiW}_{10} \mathrm{O}_{36}\left((\mathrm{RPO})_{2}\right]^{4-}\right.$. Inorg Chem, 1999, 38: 6152-6158

9 Mayer C R, Hervé, Lavanant H, et al. Hybrid cyclic dimers of divacant heteropolyanions: Synthesis, mass spectrometry (MALDI-TOF and ESI-MS) and NMR multinuclear characterisation. Eur J Inorg Chem, 2004, 2004: 973-977

10 Driss H, Boubekeur K, Debbabi M, et al. Synthesis and spectroscopic characterization of organophosphono derivatives of lindqvist niobotungstates-X-ray crystal structures of $\left(n \mathrm{Bu}_{4} \mathrm{~N}\right)_{3}\left[\mathrm{NbW}_{10} \mathrm{O}_{38}(\mathrm{RP})_{2}\right](\mathrm{R}=n \mathrm{Bu}$, Hep and Ph). Eur J Inorg Chem, 2008, 2008: 3678-3686

11 Carraro M, Modugno G, Sartorel A, et al. Optically active polyoxotungstates bearing chiral organophosphonate substituents. Eur J Inorg Chem, 2009, 2009: 5164-5174 
12 El Moll H, Dolbecq A, Marrot J, et al. A stable hybrid bisphosphonate polyoxometalate single-molecule magnet. Chem Eur J, 2012, 18: 3845-3849

13 El Moll H, Rousseau G, Dolbecq A, et al. Properties of a tunable multinuclear nickel polyoxotungstate platform. Chem Eur J, 2013, 19: 6753-6765

14 Bentaleb F, Makrygenni O, Brouri D, et al. Efficiency of polyoxometalate-based mesoporous hybrids as covalently anchored catalysts. Inorg Chem, 2015, 54: 7607-7616

15 Huo Y, Huo Z, Ma P, et al. Polyoxotungstate incorporating organotriphosphonate ligands: Synthesis, characterization, and catalytic for alkene epoxidation. Inorg Chem, 2014, 54: 406-408

16 Huo Y, Li D, Wan R, et al. Synthesis and characterization of organotriphosphonate-functionalized TM-containing polyoxotungstates. RSC Adv, 2015, 5: 106077-106082

17 Ban R, Sun X, Wang J, et al. Polyoxotungstate incorporating organotriphosphonate ligands and lanthanide ions: Syntheses, characterization, magnetism and photoluminescence properties. Dalton Trans, 2017, 46: 5856-5863

18 Kortz U, Savelieff M G, Bassil B S, et al. A large, novel polyoxotungstate: $\left[\mathrm{As}_{6} \mathrm{~W}_{65} \mathrm{O}_{217}\left(\mathrm{H}_{2} \mathrm{O}\right)_{7}\right]^{26-}$. Angew Chem Int Ed, 2001, 40: 3384-3386

19 Pradeep C P, Long D L, Cronin L. Cations in control: Crystal engineering polyoxometalate clusters using cation directed self-assembly. Dalton Trans, 2010, 39: 9443-9457

20 Knoth W H, Harlow R L. New tungstophosphates: $\mathrm{Cs}_{6} \mathrm{~W}_{5} \mathrm{P}_{2} \mathrm{O}_{23}, \mathrm{Cs}_{7} \mathrm{~W}_{10} \mathrm{PO}_{36}$, and $\mathrm{Cs}_{7} \mathrm{Na}_{2} \mathrm{~W}_{10} \mathrm{PO}_{37}$. J Am Chem Soc, 1981, 103: $1865-1867$

21 Canny J, Teze A, Thouvenot R, et al. Disubstituted tungstosilicates. 1. Synthesis, stability, and structure of the lacunary precursor polyanion $\gamma-\mathrm{SiW}_{10} \mathrm{O}_{36}{ }^{8-}$. Inorg Chem, 1986, 25: 2114-2119

22 Brown I D, Altermatt D. Bond-valence parameters obtained from a systematic analysis of the inorganic crystal structure database. Acta Crystallogr Sect B Struct Sci, 1985, 41: 244-247

23 Ritchie C, Speldrich M, Gable R W, et al. Utilizing the adaptive polyoxometalate $\left[\mathrm{As}_{2} \mathrm{~W}_{19} \mathrm{O}_{67}\left(\mathrm{H}_{2} \mathrm{O}\right)\right]^{14-}$ to support a polynuclear lanthanoid-based single-molecule magnet. Inorg Chem, 2011, 50: 7004-7014

24 Ban R, Liang Y, Ma P, et al. Synthesis, crystal structure, characterization and magnetic property of a new organophosphonate-based polyoxovanadate. Inorg Chem Commun, 2016, 71: 65-67

25 Dang $\mathrm{S}$, Yu J, Wang $\mathrm{X}$, et al. NIR-luminescence from ternary lanthanide $\left[\mathrm{Ho}^{\mathrm{III}}, \mathrm{Pr}^{\mathrm{III}}\right.$ and $\left.\mathrm{Tm}^{\mathrm{III}}\right]$ complexes with 1-(2-naphthyl)-4,4,4-trifluoro-1,3-butanedionate. J Lumin, 2011, 131: 1857-1863 


\title{
Synthesis, structure and properties of a Pr-containing organophosphonate-functionalized polyoxotungstate
}

\author{
Zongfei Yang, Pengtao Ma, Jingping Wang* \& Jingyang Niu* \\ Henan Key Laboratory of Polyoxometalate Chemistry, Institute of Molecular and Crystal Engineering, College of Chemistry and Chemical Engineering, \\ Henan University, Kaifeng 475004, China \\ *Corresponding authors, E-mail: jpwang@henu.edu.cn; jyniu@henu.edu.cn
}

Polyoxometalates (POMs), a unique class of metal-oxygen clusters with an oxygen-rich surface and high negative charge, have attracted great attention due to their unique structural variety and excellent physicochemical properties. In addition, organophosphonates, as an important kind of organic ligands with wide various and rich coordination modes, have lots of potential applications in materials, medicine and catalysis. Therefore, the synthesis of organophosphonates-based POMs with novel structure and good property is of contemporary interest. Polyoxotungstates (POTs) are viewed as one of the most important and vibrant subfamilies in POM chemistry and have been increasingly used as building blocks for the construction of systems with inorganic-organic hybrid compounds. Nevertheless, compared to the abundant derivatives of polyoxomolybdates and polyoxovanadates, organophosphonate-based POTs have been rarely studied. It is commonly that most of the structures just anchor some common transition metals $(\mathrm{Co}, \mathrm{Ni}, \mathrm{Cu})$ or even do not anchor any metal ions. In contrast, POTs incorporated with lanthanide ( $\mathrm{Ln}$ ) ions and organophosphonate ligands have been rarely explored. Up to now, only a series of Ln-containing organophosphonatebased POTs have been reported. Moreover, the $\mathrm{Ln}$ ions possess the $4 \mathrm{f}$ electrons, leading to interesting properties such as magnetic and photoluminescent properties. It appeals us to devote in investigating the interesting Ln-containing organophosphonate-functionalized POTs. Herein, a new organic-inorganic hybrid $\mathrm{CsH}\left[\mathrm{Pr}\left(\mathrm{H}_{2} \mathrm{O}\right)_{4}\left(\mathrm{AsW}_{9} \mathrm{O}_{33}\right)\left(\mathrm{W}_{2} \mathrm{O}_{3}\right)\left(\mathrm{O}_{3} \mathrm{PCOHCH}_{3} \mathrm{PO}_{3}\right) \operatorname{Pr}\left(\mathrm{H}_{2} \mathrm{O}\right)_{7}\right]_{2} \cdot 19 \mathrm{H}_{2} \mathrm{O}(\mathbf{1})$ has been successfully synthesized by reaction of $\mathrm{K}_{14}\left[\mathrm{As}_{2} \mathrm{~W}_{19} \mathrm{O}_{67}\left(\mathrm{H}_{2} \mathrm{O}\right)\right]$, HEDP and $\mathrm{PrCl}_{3} \cdot 6 \mathrm{H}_{2} \mathrm{O}$ in a conventional aqueous method. This structure was structurally characterized by single-crystal X-ray diffraction, TG analysis, PXRD and IR spectrum. The results show that compound 1 exhibits a three-dimensional (3D) framework structure which is consist of the S-shaped inorganic building block $\left\{\left[\left(\mathrm{AsW}_{9} \mathrm{O}_{33}\right)\left(\mathrm{W}_{2} \mathrm{O}_{3}\right)\right]_{2}\right\}$, four $\mathrm{Pr}$ ions and two organophosphonate groups $\left\{\mathrm{O}_{3} \mathrm{PCOHCH}_{3} \mathrm{PO}_{3}\right\}$. Variable temperature magnetic susceptibility of the solid state $\mathbf{1}$ was also measured at $1.8-300 \mathrm{~K}$ with an applied field of $1 \mathrm{kOe}$. At $300 \mathrm{~K}$, the $\chi_{\mathrm{m}} T$ product is $6.42 \mathrm{~cm}^{3} \mathrm{~K} \mathrm{~mol}^{-1}$, close to the value of $6.40 \mathrm{~cm}^{3} \mathrm{~K} \mathrm{~mol}^{-1}$ expected for four uncoupled $\operatorname{Pr}^{\mathrm{III}}$ ions $\left(L=5,3 \mathrm{H}_{4}, g=4 / 5, S=1\right)$. With cooling, the $\chi_{\mathrm{m}} T$ value decreases continuously down to a value of $5.16 \mathrm{~cm}^{3} \mathrm{~K} \mathrm{~mol}^{-1}$ at $101 \mathrm{~K}$. As the temperature further decreases, the $\chi_{\mathrm{m}} T$ value starts to decrease rapidly and reaches a minimum of $0.43 \mathrm{~cm}^{3} \mathrm{~mol}^{-1} \mathrm{~K}$ at $1.8 \mathrm{~K}$. This behavior indicates the antiferromagnetic exchange interactions and which is further confirmed by a negative Weiss constant $\theta=-39.02 \mathrm{~K}$ derived by fitting the Curie-Weiss law to the magnetic data between 68 and $300 \mathrm{~K}$ The near-infrared (NIR) emission spectra of $\mathbf{1}$ was also investigated at room temperature. Under the excitation at $448 \mathrm{~nm}$, the NIR emission spectrum of 1 consists of three characteristic peaks of the $\operatorname{Pr}^{\mathrm{III}}$ ions at $871 \mathrm{~nm}\left({ }^{\mathrm{I}} \mathrm{D}_{2} \rightarrow{ }^{3} \mathrm{~F}_{2}\right), 1017 \mathrm{~nm}\left({ }^{\mathrm{I}} \mathrm{D}_{2} \rightarrow{ }^{3} \mathrm{~F}_{4}\right)$ and $1515 \mathrm{~nm}\left({ }^{1} \mathrm{D}_{2} \rightarrow{ }^{1} \mathrm{G}_{4}\right)$. Moreover, the NIR decay curve of $\mathbf{1}$ is fitted into a second-order exponential function with lifetimes of $\tau_{1}=4.76 \mu \mathrm{s}(35.53 \%), \tau_{2}=9.71 \mu \mathrm{s}(64.47 \%)$. In the following work, other bridging ligand with different functional groups such as amino or carboxyl-functionalized organophosphonate will be introduced into this system to explore much more Ln-containing organophosphonate-based POTs with intriguing structures and properties.

polyoxometalate, organophosphonate, crystal structure, lanthanide ion

doi: 10.1360/N972018-00605 